\title{
Diversité végétale de la Forêt Classée de Yaya au Sud-Est de la Côte d'Ivoire
}

\author{
Jean-Marc Gala Bi ZAOURI ${ }^{1 *}$, Olivier N'guessan $\mathrm{YAO}^{1}$, Kafana $\mathrm{SORO}^{2}$ et \\ Dodiomon SORO ${ }^{1}$ \\ ${ }^{1}$ Université Félix Houphouët-Boigny (Cocody-Abidjan), Laboratoire des Milieux Naturels et Conservation de la \\ Biodiversité, 22 BP 582 Abidjan 22, Côte d'Tvoire. \\ ${ }^{2}$ Université NAGUI ABROGOUA, Centre de Recherche en Ecologie (CRE), 08 BP 109 Abidjan 08, Côte d'Ivoire. \\ *Auteur correspondant ; E-mail : marczaouri88@gmail.com
}

\begin{tabular}{lll}
\hline Received: 24-06-2021 & Accepted: 23-11-2021 & Published: 31-12-2021 \\
\hline
\end{tabular}

\section{RESUME}

Cette étude a été conduite afin de contribuer à une meilleure connaissance de la diversité floristique ligneuse de la forêt classée de Yaya. Au total, 50 placettes de $200 \mathrm{~m}^{2}$ ont été disposées dans les différents types d'occupation du sol. Dans chacun des rectangles, tous les individus ligneux de diamètre à hauteur de poitrine (DHP) supérieur ou égale à $10 \mathrm{~cm}$ ont été identifiés, comptés et mesurés. Ces Relevés de surfaces couplés aux inventaires itinérants ont permis d'inventorier 167 espèces rangées dans 134 genres que se partagent 47 familles. Les familles les plus importantes dans l'ordre étaient : les Fabaceae, les Euphorbiaceae et les Malvaceae. Les Mésophanerophytes et les mégaphanerophytes étaient dominants avec les pourcentages respectifs de $43 \%$ et $35 \%$. L'endozochorie était le mode de dissémination le plus rependu avec un taux de $79 \%$. 14\% des espèces recensées dans cette forêt étaient des espèces à statut particulier. Les valeurs moyennes des densités et aires basales des différents biotopes n'étaient pas statistiquement différents, qui indiquent une bonne conservation de la forêt. Ces résultats étaient donc suffisants pour justifier sa protection.

(C) 2021 International Formulae Group. All rights reserved.

Mots clés : Diversité végétale, Forêt classée de Yaya, occupation du sol, bonne conservation.

\section{Plant diversity of the Yaya Classified Forest in South-eastern Côte d'Ivoire}

\begin{abstract}
This study was conducted to contribute to a better knowledge of the woody floristic diversity of the Yaya classified forest. A total of 50 plots of $200 \mathrm{~m}^{2}$ were set up in the different land-use types. In each of the rectangles, all woody individuals with a diameter at breast height $(\mathrm{DBH})$ greater than or equal to $10 \mathrm{~cm}$ were identified, counted and measured. These area surveys coupled with the roving surveys resulted in 167 species in 134 genera shared by 47 families. The most important families in order were: Fabaceae, Euphorbiaceae and Malvaceae. Mesophanerophytes and megaphanerophytes were dominant with the respective percentages of $43 \%$ and $35 \%$. Endozory was the most common mode of spread with a rate of $79 \% .14 \%$ of the species recorded in this forest were special status species. The mean values of the densities and basal areas of the different biotopes were not statistically different, which indicates a good conservation of the forest. These results were therefore sufficient to justify its protection.
\end{abstract}

(C) 2021 International Formulae Group. All rights reserved.

Keywords: Plant diversity, Yaya classified forest, land use, good conservation. 


\section{INTRODUCTION}

Les forêts tropicales sont des écosystèmes extrêmement utiles et précieux qui jouent un rôle primordial dans la stabilisation du climat (Adjakpa et al., 2013). Elles sont les écosystèmes végétaux les plus complexes et les plus diversifiés de la planète, au point qu'un nombre important d'entre elles fait partie des « points chauds » de la biodiversité mondiale et constitue une priorité en matière de conservation de la nature (Myers et al., 2000). Ces forêts jouent un rôle important dans le stockage du carbone alors que celui-ci représente un danger très grave pour l'atmosphère (Koffi, 2016). Elles protègent également les sols contre l'érosion, améliorent le microclimat en réduisant la température au niveau du sol et assurent le maintien des qualités nutritives du sol. En dépit de leur importance, ces forêts subissent une forte pression humaine qui constitue aujourd'hui une menace pour la pérennisation des biens et services qu'elles fournissent.

En Côte d'Ivoire, la dégradation du couvert végétal est particulièrement préoccupante. Le rythme de destruction qui est de 265000 ha de 1990 à 2000 est parmi les plus élevés du monde (Achard et al., 2002). La dégradation de la biodiversité est due majoritairement à la pression démographique, aux pratiques agricoles et aux exploitations forestières et secondairement aux prélèvements des plantes médicinales (Adingra, 2017). En effet, il est essentiel de comprendre tous les déterminants de la diversité végétale actuelle pour ces écosystèmes qui figurent parmi les plus menacés de la planète. Selon Kouassi et al. (2009), on ne peut pas aborder l'étude des mécanismes qui contrôlent les divers aspects d'une biocénose et espérer en comprendre le déterminisme, si l'on ne connait pas suffisamment les composantes. Contrairement aux autres forêts tropicales, l'énorme avantage de la végétation ivoirienne est d'avoir été bien étudiée (Adingra et N'dja, 2016) Cependant certaines formations végétales sont encore peu étudiées comme celle de la Forêt Classée de Yaya (Alépé). Cette forêt classée mérite une attention particulière car elle figure parmi les derniers massifs de Côte d'Ivoire les mieux conservés et dont la survie est compromise par la pression démographique. L'objectif de ce travail est de contribuer à une meilleure connaissance de la diversité floristique ligneuse de la Forêt Classée de Yaya.

\section{MATERIEL ET METHODES Milieu d'étude}

La forêt classée de Yaya est localisé entre $5^{\circ} 36$ et $5^{\circ} 49$ de latitude nord et entre $03^{\circ} 41$ et $03^{\circ} 27$ de longitude ouest (Figure 1). Elle est située dans le sud-est de la Côte d'Ivoire, dans la région de la Mé et dans la Sous-Préfecture de Oghlawapo et s'étend sur 23879 ha. Il s'agit d'une forêt dense semidécidue soumise à un climat sub-tropical subhumide, appartenant au secteur mésophile d'après les subdivisions établies par Guillaumet et Adjanohoun (1971). La végétation climacique est la forêt dense humide semidécidue au sens d'Aubreville (1947). La température moyenne annuelle est de $26,5^{\circ} \mathrm{C}$, avec une amplitude faible de 3 à 4 . La pluviométrie moyenne annuelle est comprise entre 1200 et $1700 \mathrm{~mm}$.

\section{Collecte des données}

À partir de la carte d'occupation du sol de la Forêt Classée de Yaya complétée par des prospections de terrain, des sites de relevé de végétation ont été aléatoirement installés dans des unités de végétation floristiquement homogènes. Le plan d'échantillonnage a été conçu de sorte à inventorier les différents types de formations végétales. Des placettes rectangulaires ont été disposées dans la forêt dégradée ( $\mathrm{n}=3$ placettes), dans la forêt dense $(\mathrm{n}=41$ placettes $)$ et dans les zones de reboisement ( $\mathrm{n}=6$ placettes), le nombre de placettes d'échantillonnage varie en fonction de la superficie de chaque type d'occupation du sol. La taille des relevés était de $2000 \mathrm{~m}^{2}$. Une telle surface permettra de prendre en compte les aires minimales des communautés végétales (Senterre, 2005). Dans chacun des rectangles, tous les individus ligneux de diamètre à hauteur 
de poitrine (DHP) supérieur ou égale à $10 \mathrm{~cm}$ sont identifiés, comptés et mesurés.

L'inventaire floristique a consisté à dresser la liste exhaustive de toutes les espèces ligneuses présentes dans la surface-échantillon selon les principes de la phytosociologie (Gillet et al., 1991). Des inventaires itinérants complémentaires ont été menés dans toutes les parties de la forêt y compris dans les zones anciennement cultivées. Ces inventaires ont permis de compléter la liste floristique. La nomenclature qui a été adoptée est celle de Lebrun et Stork (1991-1997). Celle des familles suit APG III (2009).

\section{Analyse des données}

Un catalogue floristique a été établi. Il était constitué de toutes les espèces identifiées dans les 50 relevés de terrains auxquelles les espèces supplémentaires rencontrées lors des relevés itinérants ont été adjointes. Afin d'avoir une idée globale de la richesse, de la composition floristique et de la structure de la végétation, le nombre d'espèces, de genres et de familles a été déterminé. Par la suite, la répartition phytosociologique et les types biologiques ont été complétés en référence à Raunkiaer (1934). Par confrontation de notre liste à celles d'Aké-Assi (1998) et de l'UICN (2015), les espèces à statut particulier figurant sur la liste floristique ont été mises en évidence. La diversité quantitative des biotopes a été évaluée par le calcul de certains indices, tels que l'indice de diversité de Shannon, qui constitue avec l'indice d'importance des familles, les deux indices les plus utilisés en Côte d'Ivoire (Adou Yao, 2005). L'indice de Shannon peut être appliqué aussi bien aux populations arborées, arbustives et herbacées, il se calcule selon la formule suivante :

\section{$H^{\prime}=-\sum[(n i / N) x \ln (n i / N)]$}

Avec $\mathrm{Ni}=$ nombre d'individus de l'espèce $\mathrm{i}$; $\mathrm{N}=$ nombre total d'individus inventoriés dans les placeaux. L'indice d'équitabilité de Piélou qui est encore appelé indice de régularité ou d'équirépartition a été également utilisé. Il traduit la manière dont les individus sont distribués à travers les espèces (Adjakpa et al., 2013). Il permet d'apprécier la répartition des différentes espèces dans un biotope donné. Il varie entre 0 et 1 . Il traduit également la dominance d'une quelconque espèce dans l'espace étudiée et se calcule selon la formule suivante :

$$
E=H^{\prime} / \ln S
$$

Dans cette formule, E désigne l'indice d'équitabilité de Piélou, $\mathrm{H}$ ' est l'indice de Shannon et $\mathrm{S}$ représente le nombre total d'espèces de la parcelle ou de l'espace concerné. Afin de calculer la similitude entre les biotopes de la Forêt Classée de Yaya, un diagramme de Venn synthétique a été réalisé, Il a été fait à l'aide de l'outil génomique bioinformatique et évolutif via le lien: http://bioinformatics.psb.ugent.be/webtools/ve $\mathrm{nn} /$. Le diagramme de venn aussi appelé diagramme logique a permis d'illustrer les relations simples entre les différents biotopes (Vroh et al., 2017). Des tests de comparaison de moyennes ont été effectués afin de faire des corrélations entre certains des paramètres calculés. Le but de l'analyse est de vérifier si les moyennes des valeurs mesurées dans les différents groupes, sont significativement différentes. Avant de faire ces comparaisons, la normalité de la distribution des données a été vérifiée par le test de Shapiro-Wilk. Dans notre cas, cette distribution ne respectant pas la normalité, même après transformation, le test de Kruskal-Wallis a été choisi. Il s'agit d'un test non paramétrique à un facteur. Le niveau de significativité choisi pour ces analyses, est de $5 \%$. Chaque fois que la probabilité calculée était significative, le test de Dunn a été effectué afin de comparer deux à deux les moyennes et apprécier les différences significatives qui existent. 


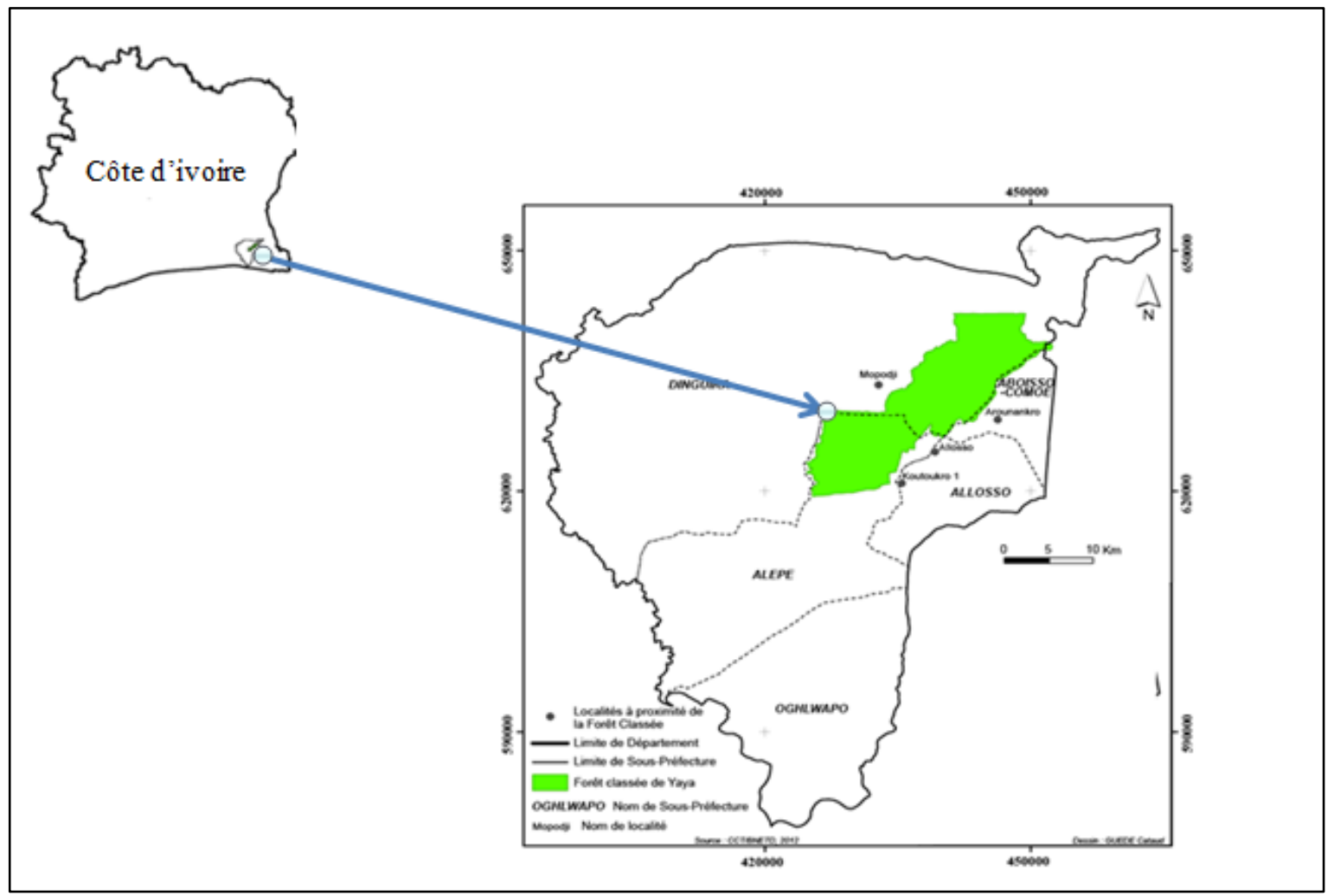

Figure 1 : Localisation de la zone d'étude.

\section{RESULTATS}

\section{Composition floristique}

Dans l'ensemble de la forêt classée, 9967 individus ligneux repartis en 167 espèces rangés dans 134 genres que se partagent 47 familles ont été recensés. La famille des Fabaceae, avec 28 espèces soit un taux de $28 \%$, était la plus représentée (Figure 2). Elle était suivie des familles des Euphorbiaceae et des Malvaceae qui avaient chacune 12 espèces, soit $11 \%$. Les autres familles dominantes étaient les Meliaceae, les Rubiaceae et les Sapotaceae (8 espèces soit $7 \%$ chacune), les Moraceae (7 espèces soit $6 \%$ ), les Clusiaceae et les Annonaceae ( 6 espèces soit $5 \%$ chacune) et les Apocynaceae (5 espèces soit $4 \%$ ).Parmi les genres que comptait la forêt classée, les plus représentés étaient le genre Diospyros (4 espèces), les genres Ficus, Garcinia,
Maranthes, Trichilia et Trichoscypha comptaient 3 espèces chacun. En ce qui concerne les différents biotopes, les forêts denses étaient les plus riches (Tableau 1). Elles comprenaient 162 espèces reparties en 130 genres rangés dans 47 familles. Ces forêts denses étaient dominées par la famille des Fabaceae (24 espèces) suivie des familles des Euphorbiaceae (11 espèces), des Malvaceae (11 espèces) et des Sapotaceae (8 espèces). Quant à la zone de reboisement, elle comportait 117 espèces reparties en 99 genres que comportaient 39 familles botaniques. Elle était dominée par la famille des Fabaceae (19 espèces) suivie de celles des Malvaceae (9 espèces), des Euphorbiaceae (8 espèces) et des Rubiaceae ( 7 espèces). Les forêts dégradées constituaient le biotope le moins diversifié. Elles comprenaient 95 espèces ligneuses 
reparties en 82 genres au sein de 35 familles. Cette partie de la forêt était dominée par la famille des Fabaceae qui comptait 19 espèces. Elle était suivie des Euphorbiaceae (6 espèces), des Clusiaceae (6 espèces) et des Apocynaceae (3 espèces).

\section{Types biologiques et mode de dispersion des diaspores \\ Types biologiques}

Dans l'ensemble de la forêt classée, les mésophanérophytes (Figure 3) étaient dominants avec 72 espèces soit une proportion de $43 \%$. Elles étaient suivies des microphanérophytes avec 60 espèces soit une proportion de $35 \%$. Les mégaphanérophytes et les nanophanérophytes étaient les moins représentés avec des proportions respectives de $21 \%$ et de $1 \%$. Quatre (4) types biologiques ont été inventoriés dans les 3 types de formations forestières, ce sont les (Figure 4). Dans la zone de forêt dense, les mésophanérophytes et les microphanérophytes dominaient avec des proportions respectives de $44 \%$ et $34 \%$, venaient les mégaphanérophytes avec une proportion de $24 \%$. Les nanophanérophytes étaient également moins représentés avec une proportion de $1 \%$. Dans les zones de reboisement, les microphanérophytes et les mégaphanérophytes dominaient avec des proportions respectives de $47 \%$ et $32 \%$, elles étaient suivies des mésophanérophytes avec une proportion de $21 \%$. Les nanophanérophytes n'étaient pas représentés. Au sein des forêts dégradées, deux types biologiques ont été enregistrés. C'étaient les mésophanérophytes et les microphanérophytes avec des proportions respectives de $25 \%$ et $75 \%$. Les mégaphanérophytes et les nanophanérophytes n'étaient pas représentés.

\section{Mode de dissémination des diaspores}

$\mathrm{Au}$ cours des travaux sur les espèces ligneuses, quatre modes de dissémination ont été identifiés (Figure 5). L'endozoochorie était majoritaire avec plus de $79 \%$ des espèces inventoriées quel que soit le type de biotope. Dans la forêt dense, les espèces endozoochores dominaient et étaient suivies des espèces anémochores représentées par $16 \%$ des taxons inventoriées (Figure 6). Dans ce type de forêt, les espèces épizoochores et barochores étaient minoritaires avec des proportions respectives de $2 \%$ et $3 \%$ des taxons. Dans les zones de culture, les espèces endozoochores dominaient et étaient suivies des anémochores (14\%) puis des espèces barochores (4\%) et des épizoochores (2\%). Dans la zone de forêt dégradée, les espèces endozoochores étaient toujours en grande proportion (79\%) et les espèces épizoochores étaient minoritaires $(2 \%)$.

\section{Affinités chorologiques des espèces de la forêt}

Dans l'ensemble de la forêt classée, les espèces endémiques à la région GuinéoCongolaise (GC) étaient dominantes à plus de $80 \%$ (Figure 7). Dans la zone de forêt dense, elles représentaient $95,67 \%$ des espèces contre $4,32 \%$ pour celles faisant la transition entre les zones Guinéo-Congolaise et SoudanoZambézienne. Dans les forêts dégradées les espèces endémiques à la région GuinéoCongolaise (GC) représentaient $96,84 \%$ contre $3,15 \%$ pour les espèces de transition GuinéoCongolaise et Soudano-Zambézienne. Enfin, dans les zones de reboisement, elles représentaient $95,72 \%$ des espèces contre 4,27\% des espèces de transition GuinéoCongolaise et Soudano-Zambézienne.

\section{Indices de diversité spécifique}

$\mathrm{La}$ valeur moyenne de l'indice de Shanonn varie de $3,53 \pm 0,21$ à $3,31 \pm 0,202$ respectivement pour les zones de reboisement et les zones de forêts dégradées en passant par les forêts denses (Tableau 2). Statistiquement, tous les biotopes visités ont la même diversité $\left(\chi^{2}=4,489\right.$ et $\left.P=0,10\right)$. En ce qui concerne l'indice d'équitabilité de Piélou, il tend vers 1 sur l'ensemble des biotopes. Les valeurs varient de 0,84 dans les forêts dégradées à 0,88 dans les 
zones de reboisement (Tableau 2). Ces différentes valeurs révèlent qu'il n'existe pas de dominance d'une espèce par rapport à une autre au sein des biotopes. Statistiquement, il n'existe pas de différences significatives entre les valeurs moyennes d'équitabilité des biotopes $\left(\chi^{2}=0,82\right.$ et $\left.P=0,664\right)$.

\section{Espèces à statut particulier et à valeur commerciale de la forêt classée de Yaya}

Parmi les espèces recensées dans la Forêt Classée de Yaya, 24 espèces (soit 14\% de la richesse spécifique) sont endémiques à l'Afrique de l'Ouest (GCW). Aussi, la comparaison de la liste générale des espèces ligneuses de la Forêt Classée de Yaya avec celle de l'UICN a montré que 21 espèces ligneuses (soit $12,42 \%$ ) figurent sur la liste rouge de l'UICN (2015). Parmi celles-ci, on comptait 15 espèces à statut de conservation. Elles étaient toutes de la catégorie vulnérable. La liste floristique de la forêt comptait aussi quatre (04) espèces (soit 2,36\%) inscrites sur la liste des Plantes Rares en voie d'Extinction de AkéAssi. Il s'agissait de Garcinia afzelii Engl.; Garcinia kola Heckel; Gilletiodendron kisantuense (Vermoesen ex de Wild.) J. L et Milicia excelsa (Welw).

\section{Similitude floristique entre les différents types d'occupation du sol de la Forêt Classée de Yaya \\ Diagramme de Venn}

Selon les données issues du diagramme de Venn (Figure 8), 82 espèces étaient inféodées à la zone de forêt dégradée, 104 à la zone de reboisement et 157 espèces inféodées à la zone de forêt dense. La forêt dense et les zones de reboisement avaient deux espèces en commun en l'occurrence Bombax buonopozense P. Beauv. et Ficus capensis Thunb. La zone de forêt dégradée et la forêt dense avaient uniquement 3 espèces en commun c'étaient: Scaphopetalum amoanum A. Chev., Symphonia globulifera L.f. et Ficus mucuso Welw. Ex Ficalho. En ce qui concerne la zone reboisement et la forêt dégradée, elles comportaient le plus grand nombre d'espèces en commun c'est-à-dire 10 espèces, entre autres, Mareya micrantha (Benth.) Müll. Arg., Terminalia superba Engl. et Diels et Omphalocarpum elatum P. Beauv.

\section{Diversité structurale \\ Densité et aire basale}

La densité de tiges oscillait entre $969 \pm$ 55 tiges/ha et $1286 \pm 205$ tiges/ha (Tableau 3). Les zones de forêt dégradée en reconstitution ont enregistré la densité la plus élevée parmi tous les biotopes, elle oscillait entre $1286 \pm 205$ tiges/ha. A la suite de ce biotope, nous avons $1037 \pm 145205$ tiges/ha pour la zone de reboisement. La zone de forêt dense, avec une valeur de $969 \pm 55$ tiges/ha, détenait la densité la plus faible. Statistiquement la différence de densité entre les biotopes n'est pas significative $\left(\mathrm{X}^{2}\right.$ et $\left.\mathrm{P}\right)$. Quant à l'aire basale, les valeurs variaient de $23,23 \pm 15,27 \mathrm{~m}^{2} /$ ha à $34,06 \pm 19,9$ $\mathrm{m}^{2} / \mathrm{ha}$. La zone de forêt dense a enregistré la plus grande valeur et les zones de reboisement la plus petite valeur. Quant à la zone de forêt dégradée, la valeur de l'aire basale était de $31,06 \pm 1,56$. Aucune différence significative entre les biotopes n'a été notée.

\section{Structure diamétrale}

Dans l'ensemble de la forêt classée, sept (7) classes de diamètre ont été identifiées (Figure 9). L"évolution des classes de diamètre était sensiblement identiques dans les trois formations végétales qui composaient la forêt. Cette distribution montre que ce sont les individus de la classe de $[5$ à $10 \mathrm{~cm}$ [de diamètre, qui étaient les plus représentés. Le nombre d'individus diminue quand le diamètre augmente. Les individus des classes de diamètres supérieurs à $80 \mathrm{~cm}$ étaient très faiblement représentés et n'existent pratiquement pas dans la formation végétale de forêt dégradée. 


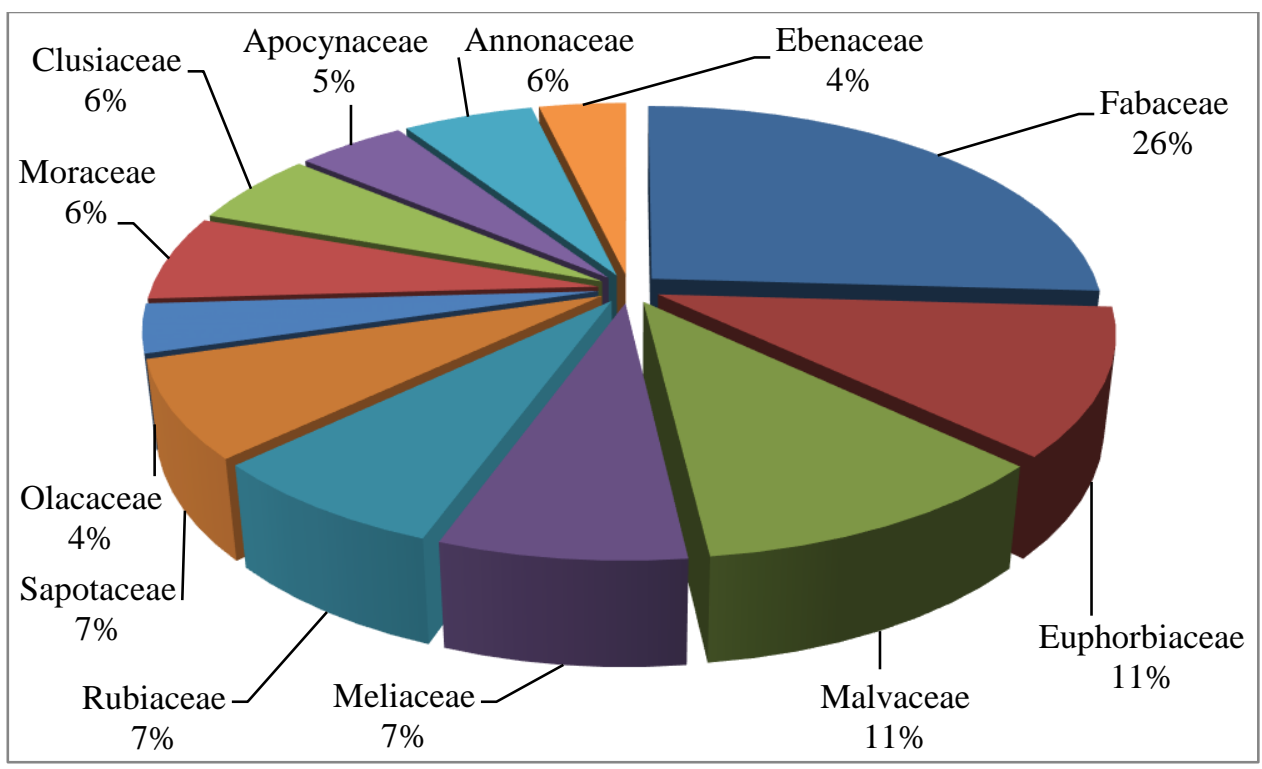

Figure 2 : Spectre des familles botaniques les mieux représentées au sein de la forêt classée de Yaya.

Tableau 1 : Richesse floristique des différentes occupations du sol de la forêt classée de Yaya.

\begin{tabular}{lccc}
\hline Biotopes & Nombre d'espèces & Nombre de genres & Nombre de familles \\
\hline Forêt dégradée & 95 & 82 & 35 \\
Forêt dense & 162 & 130 & 47 \\
Zone de reboisement & 117 & 99 & 39 \\
Total forêt classée & 167 & 134 & 47 \\
\hline
\end{tabular}

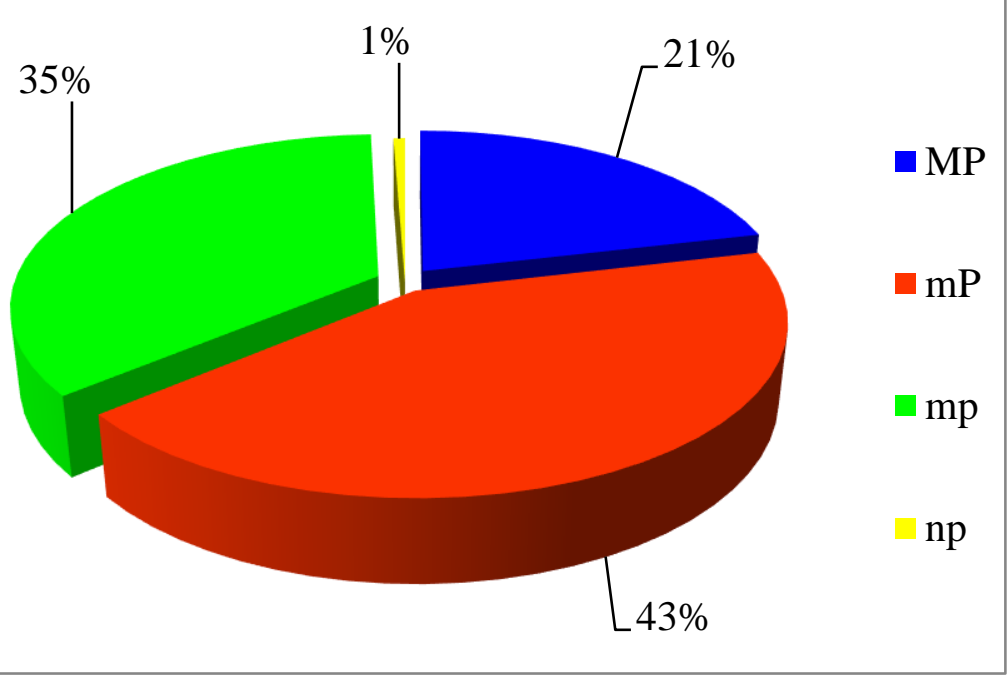

Figure 3 : Spectre des types biologiques de la flore du site de la forêt classée de Yaya. mp : microphanérophyte, $\mathbf{m P}$ : mésophanérophyte, np : nanophanérophyte, MP : mégaphanérophyte. 


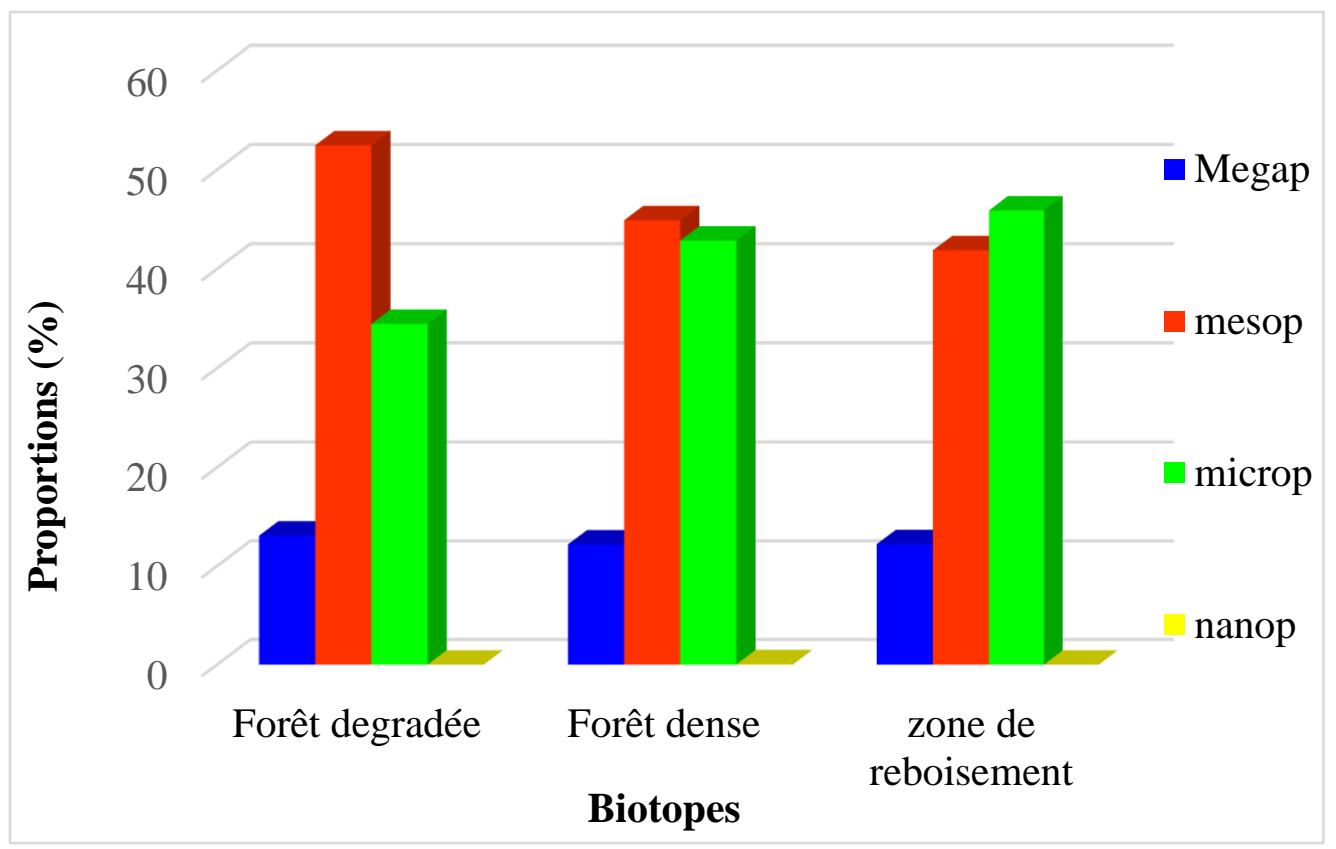

Figure 4 : Histogrammes de répartition des types biologiques dans les différentes formations végétales de la forêt classée de Yaya.

Megap : mégaphanérophyte, mesop : mésophanérophyte, microp : microphanérophyte, nanop : nanophanérophytes.

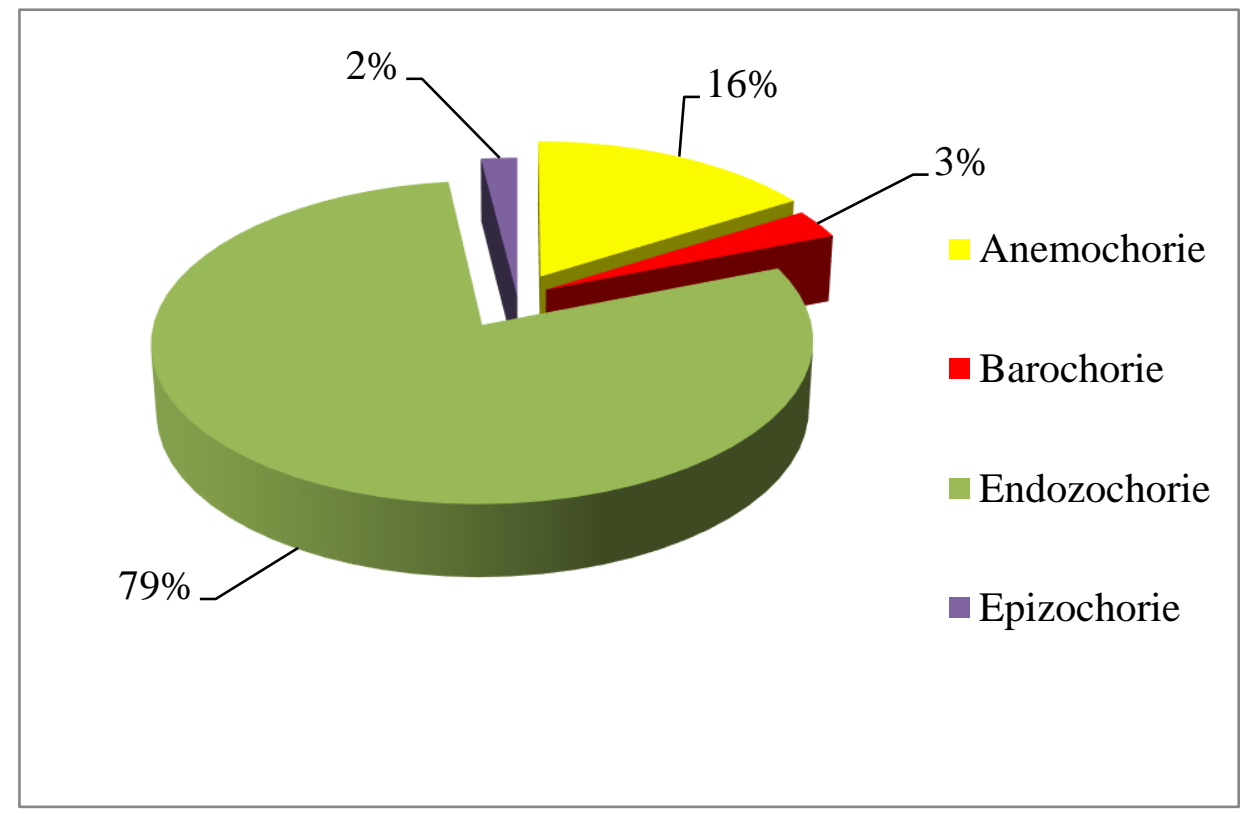

Figure 5 : Spectre des modes de dissémination des diaspores des espèces du domaine de la forêt classée de Yaya. 


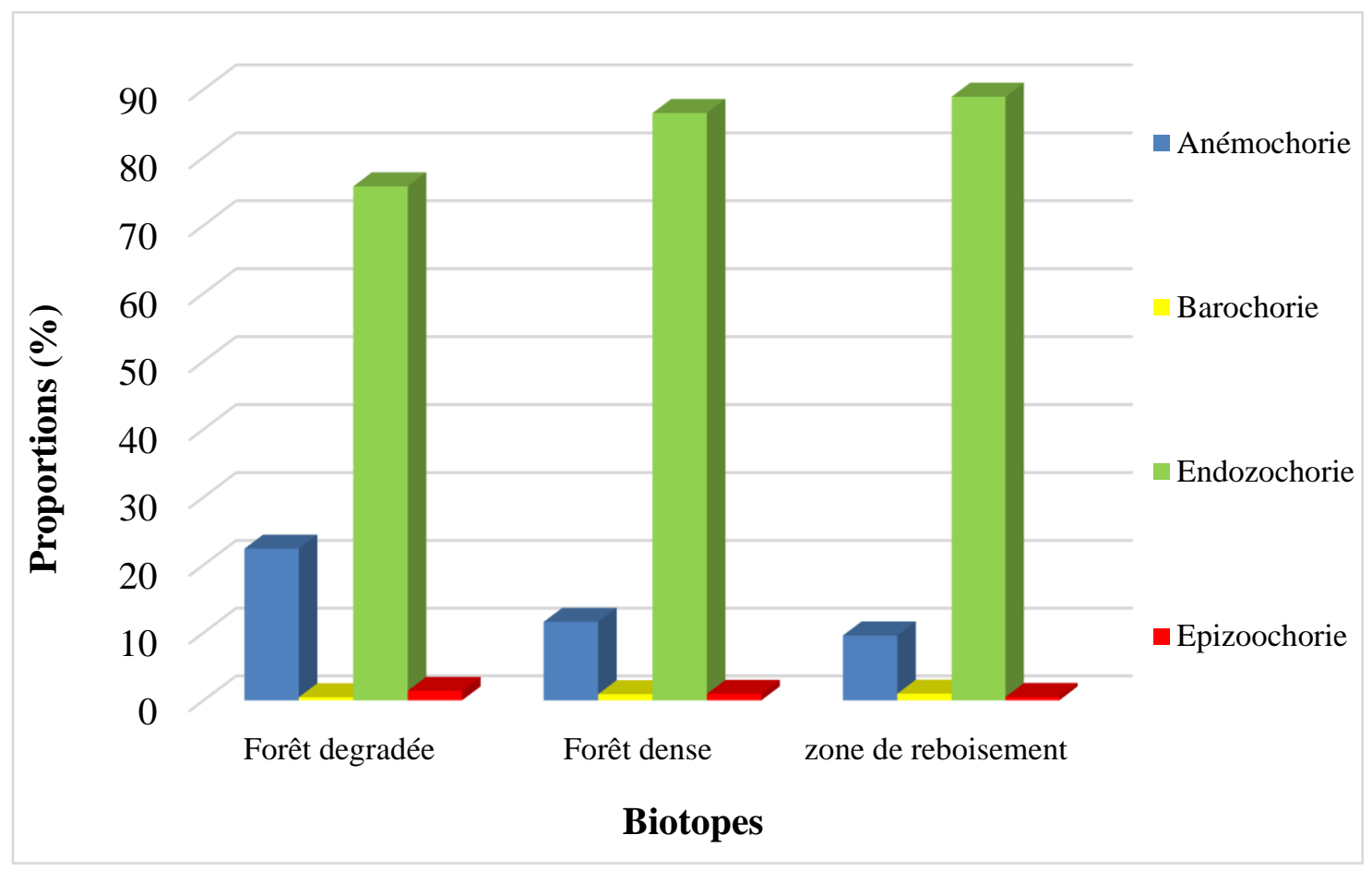

Figure 6: Histogrammes des modes de dissémination des espèces au sein des différentes formations végétales de la forêt cassée de Yaya.

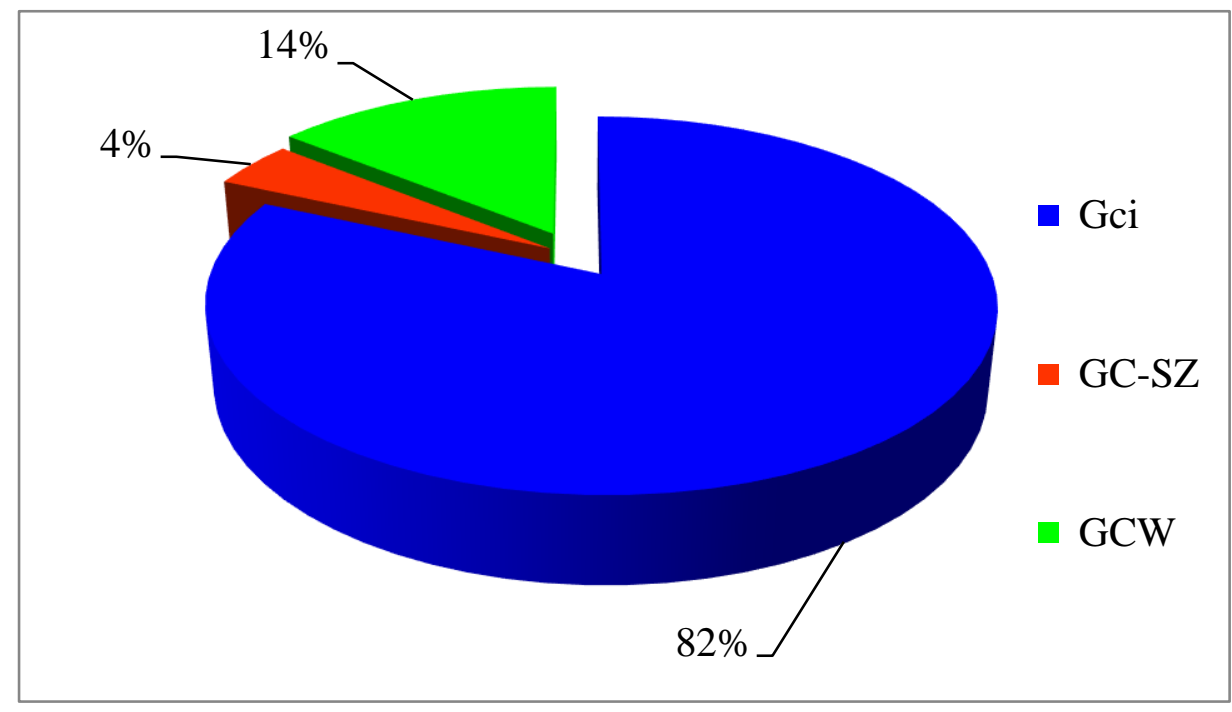

Figure 7 : Spectre des afffinités chorologiques de la Forêt classéee Yaya.

GCW : Guinéeo-Congolaises endémiques ouest-africaines ; GCi : Guinéeo-Congolaises endémiques de Côte d'Ivoire. SZ : Soudano- Zambésiennes; GC-SZ : transition GC et $\mathbf{S Z}$. 
Tableau 2 : Tableau des indices de diversité de la forêt classée de Yaya.

\begin{tabular}{lcc}
\hline Types de biotopes & Indice de Shannon & Indice de Piélou \\
\hline Forêt dense & $3,32 \pm 0,45^{\mathrm{a}}$ & $0,842 \pm 0,084^{\mathrm{a}}$ \\
\hline Forêt dégradée & $3,31 \pm 0,202^{\mathrm{a}}$ & $0,86 \pm 0,04^{\mathrm{a}}$ \\
\hline Zones de reboisement & $3,53 \pm 0,21^{\mathrm{a}}$ & $0,88 \pm 0,03^{\mathrm{a}}$ \\
\hline Statistique des tests & $\chi^{2}=4,49$ & $\chi^{2}=0,818$ \\
& $P=0,10$ & $P=0,664$ \\
\hline
\end{tabular}

Les valeurs moyennes affectées de la même lettre ne sont pas significativement différentes au seuil de 5 p.c.

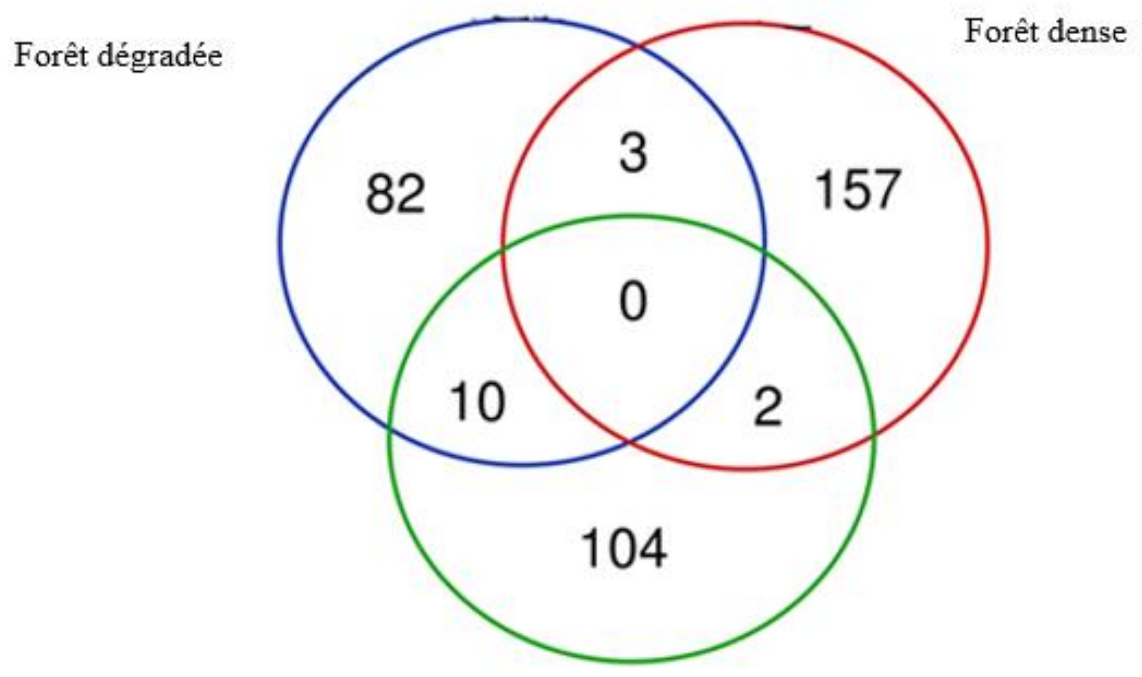

Zone de reboisement

Figure 8 : Distribution des espèces dans la Forêt Classée de Yaya suivant les différents types d'occupation du sol.

Tableau 3 : Densité et aire basale des différentes formations végétales.

\begin{tabular}{|c|c|c|}
\hline \multirow{3}{*}{ Biotopes } & $\begin{array}{l}\text { Densité } \\
\text { moyenne } \\
\text { (tiges/ha) }\end{array}$ & $\begin{array}{l}\text { Surface terrière moyenne } \\
\qquad\left(\mathbf{m}^{2} / \mathrm{ha}\right)\end{array}$ \\
\hline & \multirow[t]{2}{*}{ Anova } & Test de Kruskal-Wallis \\
\hline & & $\chi^{2}=3,32$ \\
\hline Forêt dégradée & $1286 \pm 205^{\mathrm{a}}$ & $31,06 \pm 1,56^{\mathrm{a}}$ \\
\hline Forêt dense & $969 \pm 55^{\mathrm{a}}$ & $23,23 \pm 15,27^{\mathrm{a}}$ \\
\hline Zone de reboisement & $1037 \pm 145^{\mathrm{a}}$ & $34,06 \pm 19,9^{a}$ \\
\hline
\end{tabular}




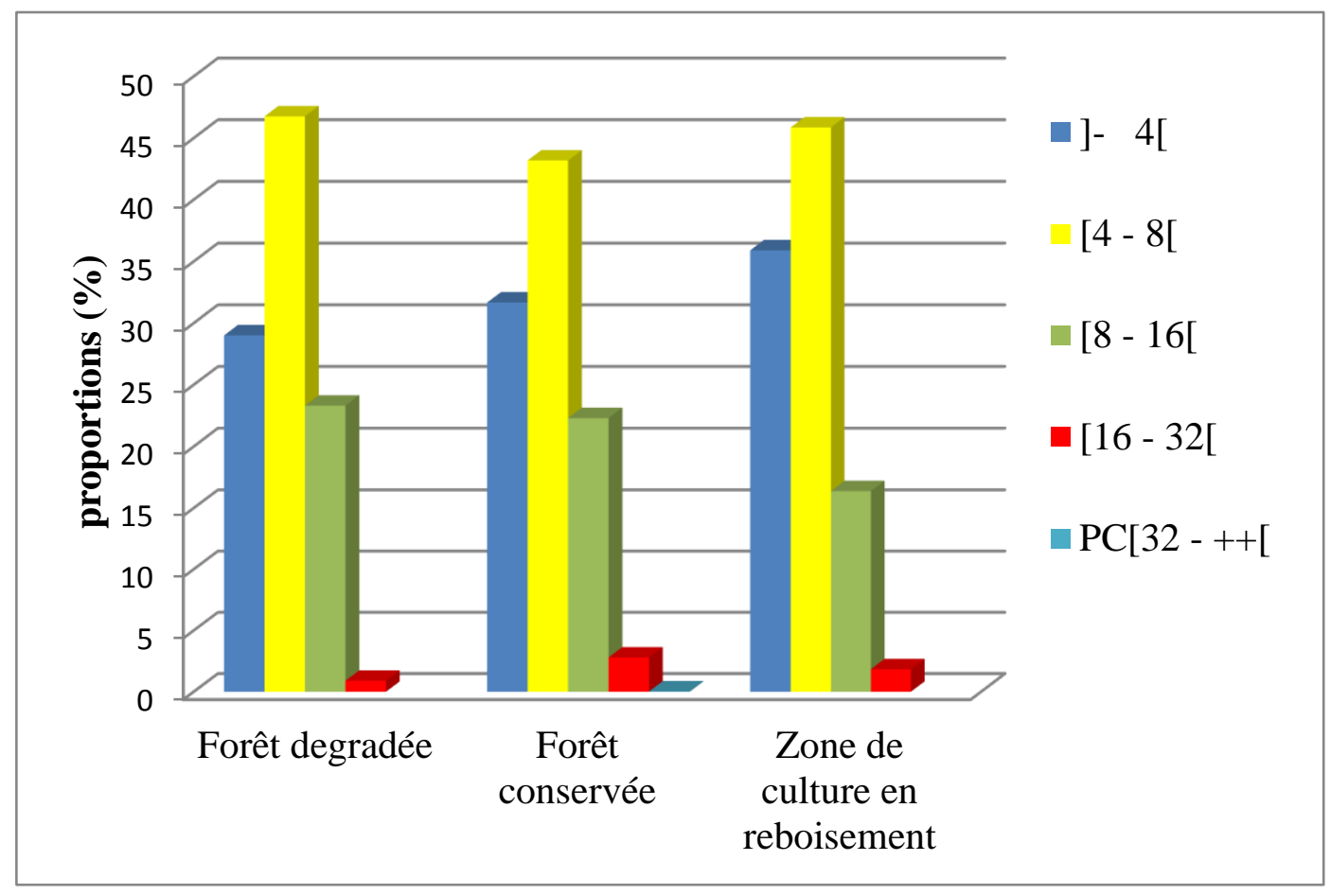

Figure 9 : Distribution des tiges par classe de diamètre dans les différentes formations végétales.

\section{DISCUSSION}

La richesse floristique de la Forêt Classée de Yaya a été estimée à 167 espèces appartenant à 134 genres rangés dans 47 familles. Cette valeur obtenue est inférieure aux valeurs observées par Koffi (2016), Adingra (2017) et N'Guessan (2018) respectivement dans le Parc National d'Azagny, les forêts classées de Bamo et d'Agbo I en Côte d'Ivoire. Cette différence serait due au fait que l'inventaire de la forêt Classée de Yaya a été axé seulement sur les espèces ligneuses alors que les auteurs cités plus haut ont pris en compte toute la diversité. Aussi, cette différence pourrait s'expliquer par le nombre de placettes installé lors de l'inventaire. Cent cinq (105) relevés phytosociologiques à l'intérieur de parcelles de $100 \mathrm{~m}$ x $20 \mathrm{~m}$ (soit $2000 \mathrm{~m}^{2}$ ) ont été effectués par (N'Guessan, 2018), 22 parcelles de 1 ha chacune ont été disposées dans le Parc National d'Azagny par (Koffi, 2016) alors que dans la forêt classée de Yaya il y a eu au total, 50 parcelles de $2000 \mathrm{~m}^{2}$, soit une superficie d'échantillonnage de 10 ha. Ces résultats montrent également que sur les 47 familles identifiées, les Fabaceae, les Euphorbiaceae, les Malvaceae, Rubiaceae, et les Meliacaeae dominent. Ces familles sont courantes dans la plupart des forêts en Côte d'Ivoire et dans les forêts africaines (Yongo, 2003). Les phanérophytes étaient les plus abondantes dans les trois types d'occupation du sol de la forêt classée de Yaya. Ces résultats ne s'écartent pas de la règle générale observée dans les forêts tropicales. Cette assertion est soutenue par Koffi (2016) qui note qu'il est de règle que dans les forêts tropicales, les phanérophytes forment la quasi-totalité du cortège floristique et que les autres types biologiques soient faiblement représentés.

Les principaux modes de dissémination étaient l'endozoochorie et l'anémochorie. Ces 
résultats concordent avec celui de N'Guessan (2018). Dans chacun des trois biotopes de la forêt, l'on note chez les espèces, une prédominance à plus de $79 \%$ des taxons endozoochores. Les fruits de ces espèces sont disséminés par les oiseaux et quelques vertébrés frugivores, notamment les antilopes, les singes, les rats, les écureuils, les éléphants. Dans le Parc National d'Azagny par exemple, les fruits représentaient $33,4 \%$ du total des espèces consommées par les éléphants (Kouamé et al., 2010). Pour Adingra (2017), la zoochorie est prépondérante dans les forêts ivoiriennes. La contribution des espèces anémochores est non négligeable. Cela est dû aux ouvertures qui ont été créées par la mise en place des cultures. En effet, l'anémochorie constitue une stratégie principale de dissémination pour les plantes des milieux ouverts (N'guessan et N'dja, 2018).

Les espèces Guinéo-Congolaises sont largement dominantes dans les trois types de forêts avec plus de 60\%. En effet, la forte proportion de ces espèces dans le cortège floristique d'une forêt, est une preuve que cette formation végétale appartient effectivement à la région Guinéo-Congolaise (N'guessan, 2018).

En ce qui concerne la densité, celle des tiges est plus élevée en zone de forêts dégradées que dans les deux autres biotopes. Les ouvertures pendant l'exploitation auraient occasionné une colonisation de ce biotope par des espèces héliophiles ce qui n'est pas le cas en forêt dense. Selon Omotoko et al. (2015), il existe une forte concurrence entre les arbres en termes de lumière et de nutriment. D'où la forte densité d'arbre en forêt dégradée qu'en forêt dense. Quant à l'aire basale, elle était plus importante en forêt dense et faible en zone de reboisement. Cette différence est surement due à la différence d'âge des individus de ces différents biotopes. Les zones de reboisement comportaient des individus juvéniles alors que la zone de forêt dense comportait des individus adultes, donc ayant une importante surface terrière. Les différents indices de diversité ont permis d'évaluer la diversité des biotopes. Les indices de diversité (Shannon et Pielou) étaient plus élevés en zone de reboisement que dans les autres biotopes. Cette diversité pourrait s'expliquer par le reboisement de nouvelles essences, l'apparition de certaines espèces suite au changement de structure du sol du fait de l'agriculture. Selon Tankoano (2017), de faibles valeurs de l'indice de Shannon ne s'observent que lorsque les sols ne laissent pousser qu'un nombre réduit d'espèces adaptées à un milieu.

\section{Conclusion}

Les résultats de l'étude qui a été menée montrent, 167 espèces réparties en 134 genres distingués dans 47 familles ont été inventoriées. Les familles les plus importantes étaient les Fabaceae, les Euphorbiaceae, les Malvaceae et les Rubiaceae. La densité des espèces était plus élevée en forêt dégradée qu'en forêt dense. Par contre l'aire basale était plus importante en forêt dense qu'en zone de reboisement. La diversité était plus élevée en zone de reboisement. Dans l'ensemble de la flore inventoriée, 21 espèces (soit 12,42\%) figuraient sur la liste rouge de l'UICN. Pour chaque classe de diamètre la forêt dense comporte le plus grand nombre d'individus. Cette étude n'ayant pris en compte que la diversité de la flore ligneuse, il serait bon, pour une meilleure connaissance de végétation de la forêt classée de Yaya, de réaliser des inventaires sur toute la flore de ce domaine forestier.

\section{CONFLIT D'INTERETS}

Les auteurs déclarent qu'ils n'ont aucun conflit d'intérêts relatif à cet article.

\section{CONTRIBUTIONS DES AUTEURS}

J-MGBZ a réalisé l'inventaire de la forêt classée de Yaya et a contribué à la rédaction du manuscrit; ONY a analysé les données de terrain et a participé à l'amélioration du manuscrit ; KS a contribué à la lecture et à 
l'amélioration du document ; DS a contribué à la lecture à l'amélioration et à la validation du manuscrit.

\section{REMERCIEMENTS}

Nous remercions les habitants du village de Kossandji et Mopodji ainsi que les habitants du campement de Zongokoi. Qui nous ont reçus chez eux durant toute la période de l'étude.

\section{REFERENCES}

Achard F, Eva HD, Stibig HJ, Mayaux P, Gallego J, Richards T, Malingreau JP. 2002. Determination of deforestation rates of the world's humid tropical forests. Science, 297: 999-1002. DOI: https://doi.10.1126/science.1070656

Adingra MMAO, N'dja JK. 2016. Diversité floristique, structure et dynamique des jachères post-culturales de la forêt classée de Bamo (Côte d'Ivoire). Agronomie Africaine, 28(1): 24-32.

Adingra MMAO. 2017. Dynamique du peuplement et stocks de carbone dans la mosaïque de végétation de la forêt classée de Bamo (Côte d'Ivoire). Thèse de Doctorat, Université Félix HouphouëtBoigny, Côte d'Ivoire, $158 \mathrm{p}$.

Adjakpa BJ, Yedomonhan H, Ahoton LE, Weesie PDM, Akpo EL. 2013. Structure et diversité floristique des îlots de forêts riveraines communautaires de la vallée de Sô du Bénin. Journal of Applied Biosciences, 65 : 4902 - 4911.

Adou YCY. 2005. Pratiques paysannes et dynamique de la biodiversité dans la forêt classée de Monogaga (Côte d'Ivoire). Thèse de Doctorat, Département Hommes, Natures et Société, Université MNHN, Paris, 233 p.

Aké-Assi L. 1998. Impact de l'exploitation forestière et du développement agricole sur la conservation de la biodiversité biologique en Côte d'Ivoire. Le Flamboyant, 46: 20-22.
APG III. 2009. An update of the Angiosperm Phylogeny Group classification for the orders and families of flowering plants. Botanical Journal of the Linnean Society, 161: 105-121. DOI: https://doi.org/10.1111/boj.12385

Aubréville A. 1947. Les brousses secondaires en Afriques Equatoriale (Côte d'Ivoire, AEF-Cameroun). Bois et Forêt des Tropiques, 2 : 24-49.

Gillet F, de Foucault B, Julve PH. 1991. La phytosociologie synusiale intégrée -objet et concepts. Candollea, 46: 315-340.

Guillaumet JL, Adjanohoun E. 1971. La végétation. In Le Milieu Naturel de la Côte d'Ivoire. ORSTOM: Paris (France) ; 157-262.

Koffi KAD. 2016. Dynamique de la végétation et valeurs de conservation des espaces anciennement cultivés du Parc National d'Azagny (sud de la Côte d'Ivoire). Thèse de Doctorat, Université Félix HouphouëtBoigny, Côte d'Ivoire, 185 p.

Kouamé D, Adou Yao CY, Nandjui A, N'Guessan KE. 2010. Le rôle de l'éléphant dans la germination des graines de Irvingia gabonensis (Irvingiaceae), Balanites wilsoniana (Balanitaceae), Parinari excelsa (Chrysobalanaceae) et Sacoglottis gabonensis (Humiriaceae) en forêt tropicale: cas du Parc National d'Azagny en Côte d'Ivoire. Int. J. Biol. Chem. Sci., 4(5): 1442-1454. DOI: 10.4314/ijbcs.v4i5.65562

Kouassi KH, N'Guessan K, Gnahoua GM, Kouassi KÉ. 2009. Flore post-culturales en zone de forêt dense semi décidue de Côte d'Ivoire. Journal of Applied Biosciences, 19: 1026-1040.

Lebrun JP, Stork AL. 1991-1997. Enumération des Plantes à Fleurs d'Afrique Tropicale. Conservatoire et Jardin Botaniques de la Ville de Genève : Genève.

Myers N, Mittermeier RA, Mittermeier CG, da Fonseca GAB, Kent J. 2000. Biodiversity hotspots for conservation priorities. 
Nature, 403: $\quad 853-858 . \quad$ DOI: https://doi.org/10.1038/35002501

N'guessan AE. 2018. Dynamique de la végétation et facteurs de reconstitution de la biomasse dans les forêts secondaires : cas de la forêt classée d'Agbo 1 (sud-est de la Côte d'Ivoire). Thèse de Doctorat, Université Félix Houphouët-Boigny, Côte d'Ivoire, $221 \mathrm{p}$.

N'guessan EA, N'dja KJ. 2018. Analyse de la diversité floristique de la forêt classée d'Agbo I (Côte d'Ivoire). European Scientific Journal, 14(9): 1857-7881. DOI:

http://dx.doi.org/10.19044/esj.2018.v14 n9p346

Omatoko J, Nshimba H, Bogaert J, Lejoly J, Shutsha R, Shaumba JP, Asimonyio J, Ngbolua KN. 2015. Études floristique et structurale des peuplements sur sols argileux à Pericopsis elata et sableux à Julbernardia seretii dans la forêt de plaine d'UMA en RDC. Journal of Innovation and Applied Studies, 13(2): 452-463.

Raunkiaer C. 1934. The Lifes Forms of Plants and Statistical Plant Geography. Oxford University Press, London.

Senterre B. 2005. Recherches méthodologiques pour la typologie de la végétation et la phytogéographie des forêts denses d'Afrique tropicale. Thèse Doctorat, Université Libre de Bruxelles, Belgique, $343 \mathrm{p}$.

Tankoano B. 2017. Contribution de la télédétection et des Systèmes d'Informations Géographiques à l'évaluation de l'impact des activités humaines sur la couverture végétale : cas du Parc National des Deux Balé (PNDB), à l'Ouest du Burkina Faso. Thèse de Doctorat, Université Nazi Boni, Burkina Faso, 111 p.

IUCN (International Union for the Concervation of the Nature). 2015. IUCN Red List of Threatened Species. Version 2015.2. IUCN, Gland, Suisse. www.iucnredlist.org.

Vroh BTA, N'Guessan KE, Adou CY. 2017. Trees species diversity in perennial crops around Yapo protected forest, Côte d'Ivoire. Academic Journal, 11(9): 98-108

Yongo OD. 2003. Contribution aux études floristiques, phytogéographiques et phytosociologiques de la forêt de N'Gotto (République de Centrafrique). Acta Botanica Gallica, 150: 119-124. 